\title{
The Research on Tests for Detecting Strength of Shallow Asphalt Pavement under Ice Frozen
}

\author{
Zhao Maocai $^{1 \mathrm{a}}$, Gao Shixian ${ }^{1 \mathrm{~b}}$, Shi Dawei ${ }^{2 \mathrm{c}}$, Huang Qishu ${ }^{2 \mathrm{~d}}$ \\ ${ }^{1}$ School of Transportation Science and Engineering, Harbin Institute of Technology, Harbin, China \\ ${ }^{2}$ Inspection Bureau of Guizhou province for traffic construction engineering quality, Guiyang, China \\ azhao_maocai@126.com, bgaoshixian0424@163.com, 'shidawei88@126.com, dhuanqs@126.com
}

Keywords: Asphalt Pavement; Penetrating Strength Detector of Shallow Pavement; Void Ratio; Freeze-Thaw Cycle; Strength of Shallow Pavement

\begin{abstract}
The thesis utilizes the Penetrating Strength Detector of Shallow Pavement to conduct impacting penetration experiment, uniaxial compression test and diametral compression test to asphalt mixture of different void ratios and cycles of freezing and thawing, and then we've obtained the relationship between average penetration depth and compressive strength as well as tensile splitting strength of mixture, moreover, the initial reference standard of the Penetrating Strength Detector of Shallow Pavement is established. Afterwards, the thesis conducts impacting penetration test to the asphalt pavement cores from Chongzun freeway in Guizhou Province suffering from ice frozen damage. The effectiveness of the Penetrating Strength Detector of Shallow Pavement has been proved.
\end{abstract}

\section{Introduction}

Nowadays, the on-the-spot detective methods involving strength of shallow pavement conclude Benkelman Beam, Falling Weight Deflectometer(FWD), Ground Penetrating Radar, Ultrasonic wave method, WINDSOR method, Needle penetrating method of concrete (Penetrating resistance method and Detective needle pressing-in method of concrete strength) Rebound Apparatus, etc.; The methods in laboratory conclude CBR method, compression and tensile splitting tests of core samples, etc[1-3]. Generally speaking, the detected deflection is the comprehensive response of all structure layers under pavement surface to loading. Therefore, they can't detect the strength of a certain depth in pavement surface layer [4].

Ground Penetrating Radar is used to detect the thickness of highway surface course and defects. Because the damage from ice frozen to shallow pavement doesn't have obvious interface, it can not detect the strength of a certain depth in pavement surface layer, either [5-7]. Ultrasonic wave method is similar with Ground Penetrating Radar, which also can't detect the strength of a certain depth in surface layer [8].

What has Rebound Apparatus detected is a comprehensive value of concrete strength in a certain depth of concrete surface. The Rebound Apparatus can only obtain a overall evaluation instead of concrete strength of different depths [9].

WINDSOR method, as known as Needle penetrating method of concrete (Penetrating resistance method and Detective needle pressing-in method of concrete strength), is able to evaluate concrete strength with penetration depth or pressing-in depth according to corresponding relationship between penetration depth and concrete strength given by ASTM[10]. However, this method to evaluate the damage of shallow pavement under ice frozen still exist deficiencies as follows: 
a. Auxiliary device to provide counterforce needs to be fixed on spot in advance, which is tedious and inconvenient;

b. The concrete strength of a defined depth can't be detected;

c. The penetration work can't be controlled. In most situations, after penetration work is fixed, the depth of penetrator injecting-in or pressing-in can't be controlled artificially (mainly affected by concrete strength and magnitude of penetration work - static pressure).

In consequence, the author has designed the Penetrating Strength Detector of Shallow Pavement which can overcome the detection deficiency above, aiming at resolving the detection and evaluation problem of shallow pavement under ice frozen. This detector is able to evaluate the strength of shallow pavement rapidly according to the relationship between detection data and multiple mechanical properties of pavement established by relevant experiments, possessing comprehensive applicability and extensive prospect.

\section{The changing regularity about compressive strength and tensile splitting strength of the asphalt mixture in different void ratios}

The experiment adopts similar materials and asphalt-aggregate ratio with Chongzun freeway pavement in Guizhou and molds several specimens through rotation compacting method according to Superpave specification, afterwards, the linear contrast relationship between void ratios and quality is obtained by fitting. Therefore, the specimen quality corresponding to design void ratios of $3 \%, 6 \%, 9 \%$ and $12 \%$ can be obtained, then we molds 36 specimens with 9 in each group corresponding to 4 void ratios according to the relationship above through rotation compacting method again.

We select 3 specimens from each group and put them in thermostatic water tank of $25^{\circ} \mathrm{C}$ together for heat insulation of 4 hours, then the experiments are conducted on MTS material testing machine with loading speed of $2 \mathrm{~mm} / \mathrm{min}$, afterwards the compressive strength and tensile splitting strength are obtained respectively.

The relationship that the compressive strength and tensile splitting strength obtained from experiments varies with void ratios is shown as Figure 1 and 2.

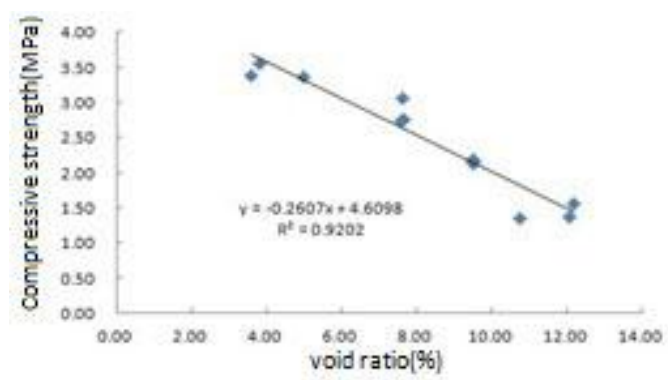

Figure 1. Change of compression strength with voidage

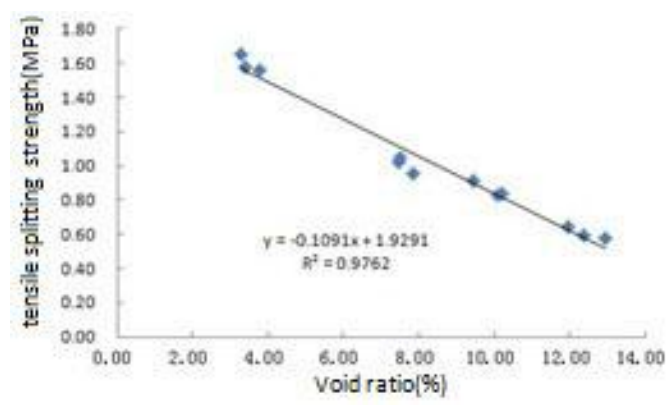

Figure 2. Change of splitting strength with voidage 
As we can see from the two Figures above, the compressive strength and tensile splitting strength of asphalt mixture decrease as void ratio grows. The two pairs of relationship above own a great relativity by regression and the regression coefficient reaches 0.9209 and 0.9762 respectively. The slope of straight lines obtained by regression in the Figures is relatively large, which means that void ratio has great influence on strength of asphalt mixture.

\section{The impacting penetration test to asphalt mixture of different void ratio}

The experiment adopts the self-designed Penetrating Strength Detector of Shallow Pavement(10kg, 8mm) to conduct impacting penetration experiment under room temperature $\left(25^{\circ} \mathrm{C}\right)$, and testing targets are the remaining specimens molded. Because the testing specimens are independent without peripheral pressure, the thesis has manufactured a fixture of detecting asphalt mixture specimens to simulate the peripheral pressure.

The testing results of impacting penetration experiment to 12 specimens with different void ratios are shown as Figure 3 and 4.

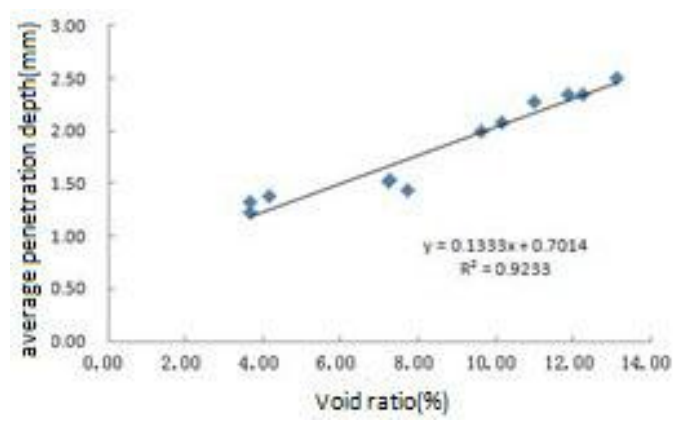

Figure 3. Change of peening times with voidage

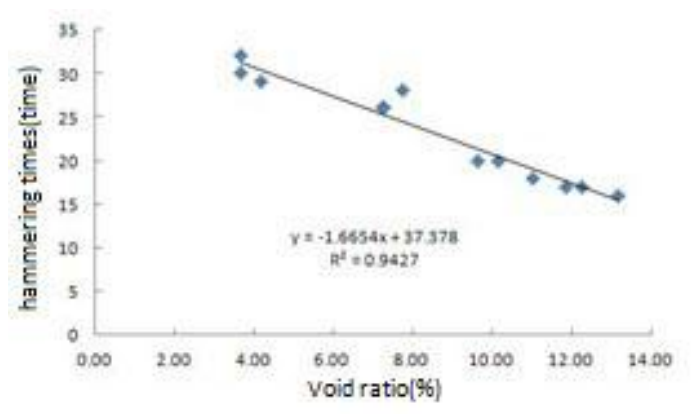

Figure 4. Change of peening times with voidage

The linear relationship between void ratio and average penetration depth as well as hammering times is good as the Figure4 have shown which enables us to contact the average penetration depth with the compressive strength and tensile splitting strength of asphalt mixture through the easily available and important index-void ratio.

The contrast analysis between impacting penetration experiment and compressive strength as well as tensile splitting strength of asphalt mixture

Firstly, we obtained the following equations through linear fitting to the experimental data:

$$
\begin{aligned}
& \mathrm{Rc}=-0.2607 \mathrm{VV}+4.6098 \\
& \mathrm{RT}=-0.1091 \mathrm{VV}+1.9291 \\
& \mathrm{Da}=0.1333 \mathrm{VV}+0.7014 \\
& \mathrm{~N}=-1.6654 \mathrm{VV}+37.378
\end{aligned}
$$

Where Rc is compressive strength,(MPa), RT is tensile splitting strength,(MPa), Da is average penetration depth, $(\mathrm{mm}), \mathrm{N}$ is hammering times and $\mathrm{VV}$ is void ratio, $(\%)$. 
We can obtain the relationships between compressive strength, tensile splitting strength as well as void ratio and average penetration depth as well as hammering times respectively according to the equations (1-4), which are shown as follows:

$$
\begin{aligned}
& \mathrm{Rc}=-1.9557 \mathrm{Da}+5.9816 \\
& \mathrm{RT}=-0.8185 \mathrm{Da}+2.5032 \\
& \mathrm{VV}=7.5019 \mathrm{Da}-5.2618 \\
& \mathrm{Rc}=0.1565 \mathrm{~N}-1.2413 \\
& \mathrm{RT}=0.0655 \mathrm{~N}-0.5195 \\
& \mathrm{VV}=-0.6005 \mathrm{~N}+22.4439
\end{aligned}
$$

Considering the uniformity of asphalt mixture specimens prepared in laboratory and the testing results of impacting penetration experiment, it's found that the average penetration depth shows linear change with the hammering times. Therefore, the thesis choose average penetration depth as the detection index of Penetrating Strength Detector of Shallow Pavement, while hammering times can only be the detection referenced index at the situation that total penetration depth is $10 \mathrm{~cm}$.

\section{The changing regularity of asphalt mixture's tensile splitting strength in room temperature after freezing and thawing}

Considering the climate features in Guizhou and existing conditions in laboratory, the thesis measures tensile splitting strength of the specimens, defining freezing for 8 hours and thawing for 16 hours as a freezing and thawing cycle, then 3 cycles as a small cycle.

We can obtain the relationship curve of tensile splitting strength of the asphalt mixture specimens changing with freezing and thawing cycles by tensile splitting strength experiment, which is shown in Figure 5.

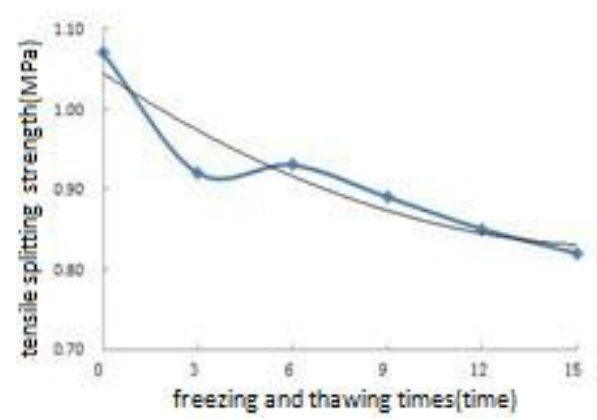

Figure 5. Change of tensile splitting strength after freeze-thaw cycles

As we can see from the Figure above, the tensile splitting strength of asphalt mixture in room temperature tends to decrease with freezing and thawing cycles grow. The tensile splitting strength varies obviously after 3 freezing and thawing cycles and then tends to ease up, the reason of which is the porosity characteristic of asphalt mixture. After vacuum water-saturated test, the inner void of mixture is filled with water, and then the void osmotic pressure and expansive force of ice are generated during water in voids turning into ice, which makes the void in specimens grow rapidly and tensile splitting strength of the first 3 freezing and thawing cycles decrease sharply. While after several cycles, the removal quantity of water during freezing process goes down gradually and the expansive force also tends to be steady, so the change of tensile splitting strength tends to ease up. 


\section{The impacting penetration experiment of asphalt mixture after freezing and thawing}

In order to obtain the relationship between testing results of the Penetrating Strength Detector of Shallow Pavement and tensile splitting strength of asphalt mixture under different times of freezing and thawing cycles, the thesis conducts impacting penetration experiment to the same specimens molded and obtains the changing curve of average penetration depth varying with freezing and thawing times under different freezing and thawing damage, shown in Figure 6.

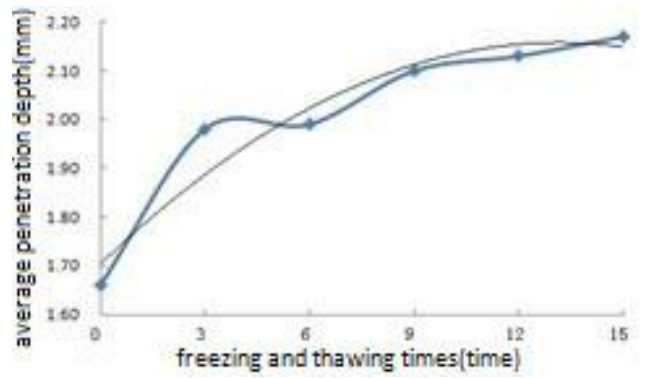

Figure 6. Change of average penetration depth with freeze-thaw cycles

As we can see from the Figure above, the average penetration depth tends to increase as the freezing and thawing times grow, and it also represents the behavior that the curve varies obviously in the first 3 freezing and thawing cycles and then tends to ease up afterwards, whose reason is almost the same with the former tensile splitting experiment.

\section{The contrast analysis between impacting penetration experiment and tensile splitting strength experiment under freezing and thawing}

After fitting analysis with the testing data, we can calculate to obtain the relationship between tensile splitting strength of asphalt mixture and average penetration depth of the Penetrating Strength Detector of Shallow Pavement, shown in Figure 7.

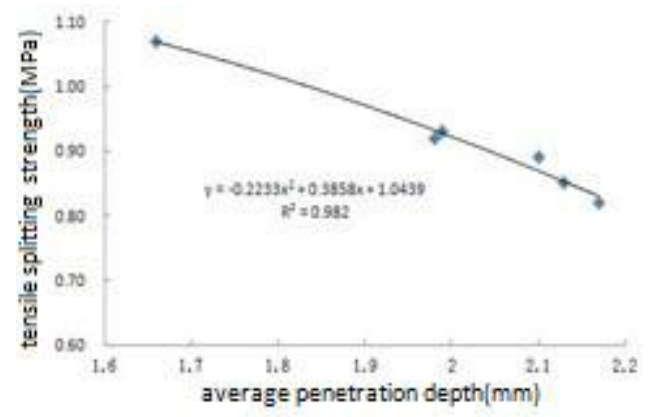

Figure 7. Relationship between tensile splitting strength and average penetration depth

The average penetration depth grows from $1.66 \mathrm{~mm}$ to $2.17 \mathrm{~mm}$, while the tensile splitting strength decreases from $1.07 \mathrm{MPa}$ to $0.82 \mathrm{MPa}$ along with it. The regression parameter $\mathrm{R} 2$ can reach 0.9820 after fitting.

\section{The evaluation to compressive strength and tensile splitting strength of the core samples}

After analyzing the data above, the overall compressive strength level of core samples is relatively low and compressive strength of $60 \%$ core samples is below 3MPa. The tensile splitting strength mainly lies in the range of $0.6-1.5 \mathrm{MPa}$, which can satisfy the specification requirement basically. The void ratio of nearly a half of core samples lies in 5\%-9\%, and some core samples' void ratio even reaches more than $13 \%$. As the Technical Specification for Construction of Highway Asphalt Pavement has demanded, the void ratio shall be kept between 3\%-5\%. Therefore only $12 \%$ 
of the core samples can meet the requirement of specification, which demonstrates that the asphalt pavement of Chongzun freeway has been damaged badly under long-term freezing and thawing and other complex conditions.

\section{Conclusion}

According to the actual testing contrast, the combination of falling hammer weighing $10 \mathrm{~kg}$ and penetrator with diameter of $8 \mathrm{~mm}$ is determined as detection components.

The changes of asphalt mixture specimens' mechanical property under different freezing and thawing times are controlled by designing different void ratios, and we conduct impacting penetration test, uniaxial compression test and tensile splitting test to the specimens and then obtained the conclusion that compressive strength and tensile splitting strength of asphalt mixture both decrease as average penetration depth in impacting penetration test increases, while void ratio increases as average penetration depth increases. We can establish the initial reference standard of the Penetrating Strength Detector of Shallow Pavement for detecting the void ratio, compressive strength and tensile splitting strength in room temperature by linear fitting.

The thesis conducts impacting penetration experiment to the core samples in asphalt pavement of Chongzun freeway in Guizhou Province under ice frozen damage, and then we obtained the void ratio, compressive strength and tensile splitting strength of the core samples in depth of $0-100 \mathrm{~mm}$ by utilizing the detection reference standard established formerly. We have offered a great evaluation on the degree of the pavement suffering from ice frozen damage and also provided a reliable reference for the follow-up maintenance work.

\section{Summary}

This research is sponsored by the department of China science ministry No: 2008BAG10B02.

\section{References:}

[1] Liu Huaqing, Han Jiguang. Research on Nondestructive Detection Techniques of Freeway Pavement[J]. Journal of Highway and Transportation Research and Development, 2009,(1):49-50.

[2] Hu Xiaguang. The Present Status and Development of Rapid Detection Techniques of Pavement at Home and Abroad[J]. Journal of China and Foreign Highway, 2003, 23(6):95-99.

[3] Wang Zehua. Research on Nondestructive Detection Techniques of Pavement[J]. Journal of Anhui University Architecture, 2006, 14(2):22-24.

[4] ASTM. Standard Test Method for Measuring Deflections with a Light Weight Deflectometer (LWD) E2583 - 07[S] 2007.

[5] Samer Lahouar, Imad L.AI-Qadi. Automatic Detection of Multiple Pavement Layers from GPR Data[J]. NDT\&E International 41(2008):69-81.

[6] Timo Saarenketo, Tom Scullion. Road Evaluation With Ground Penetrating Radar[J]. Journal of Applied Geophysics, 43_2000:119-138.

[7] C. Fauchard, X. De'robert, J. Cariou. GPR Performances for Thickness Calibration on Road Test Sites[J]. NDT\&E International, 36 (2003):67-75.

[8] Zhu Jinzhang, Luo Xin, Zhao Xiaolong. Supersonic Rebound Method to Evaluate the Compressive Strength of Freeze-thaw Concrete[J]. Low Temperature Architecture Technology, 2005(3):3-5.

[9] Natianal Standard of the People's Republic of China. Technical Specification for Inspection of Concrete Compressive Strength by Rebound Method[S]. JGJ/T23-2001.

[10] ASTM. Standard Test Method for Penetration Resistance of Hardened Concrete C803 / C803M - 03(2008) [S]; 2008. 